\title{
Uncertainty-guided intelligent sampling strategy for high-efficiency surface measurement via free-knot $B$-spline regression modelling
}

\author{
Jian Wang ${ }^{1}$, Luca Pagani ${ }^{3}$, Liping Zhou², Xiaojun Liu², Wenlong Lu ${ }^{1 *}$, Richard Leach ${ }^{4}$ and Xiangqian (Jane) Jiang ${ }^{3}$ \\ ${ }^{1}$ State Key Lab of Digital Manufacturing Equipment and Technology, Huazhong University of Science and Technology, Wuhan 430074, PR China \\ ${ }^{2}$ School of Mechanical Science and Engineering, Huazhong University of Science and Technology, Wuhan 430074, PR China \\ ${ }^{3}$ The EPSRC Future Metrology Hub, University of Huddersfield, Huddersfield HD1 3DH, UK \\ ${ }^{4}$ Manufacturing Metrology Team, Faculty of Engineering, University of Nottingham, Nottingham NG8 1BB, UK \\ ${ }^{*}$ Corresponding Author: hustwenlong@mail.hust.edu.cn
}

\begin{abstract}
Intelligent sampling can be used to influence the efficiency of surface geometry measurement. With no design model information provided, reconstruction from prior sample points with a surrogate model has to be carried out iteratively, thus the next best sample point(s) can be intelligently selected. But, a lack of accurate and fast reconstruction models hinders the development of intelligent sampling techniques. In this paper, a smart surrogate model based on free-knot B-splines is used for intelligent surface sampling design with the aid of uncertainty modelling. By implementing intelligent sampling in a Cartesian, parametric or specific error space, the proposed method can be flexibly applied to reverse engineering and geometrical tolerance inspection, especially for highdynamic-range structured surfaces with sparse and sharply edged features. Extensive numerical experiments on simulated and real surface data are presented. The results show that this parametric model-based method can achieve the same or higher sampling efficiency as some recent non-parametric methods but with far less computing time cost.
\end{abstract}

Keywords: adaptive sampling; free-knot B-splines; surface reconstruction; uncertainty analysis; structured surfaces

\section{Introduction}

Sampling is an essential part of all measurement processes. As shown in Figure 1, sampling decomposes real-world continuous signals into discretised data points. Numerical characterisation of the discrete data is then conducted with a computer [1,2]. With a dense and uniform sampling scheme, discretised data are normally directly processed for parametric characterisation. If sampling points are sparse and non-uniform, reconstruction of the sample data to a uniformly dense or continuous substitute form is an essential step towards a reliable measurement [3]. Specification standard ISO 25178 and $17450[4,5]$ on surface texture and form measurement recommends that a continuous substitute surface reconstruction (e.g. a dense uniform format) is the default route for surface characterisation (see the dashed flow in Figure 1). In recent years, smart sampling designs and reconstruction have been recognised as a fundamental add-in value to the cyber-physical manufacturing-oriented "industry 4.0" evolution [6].

Uniform sampling, which is well founded on Shannon's sampling and reconstruction theorems [7], is the most widely adopted strategy in surface measurement. However, recent non-uniform sampling theorems [8] have shown that uniform sampling is by no means a necessary condition for distortion-free reconstruction. It has been demonstrated that uniform sampling may lack efficiency when considering measurement time and data storage aspects. Intelligent sampling strategies, which aim to reduce sampling cost without loss of accuracy by smartly designing sample sizes or locations, has been demonstrated to be more efficient than uniform sampling in many applications [9-11].

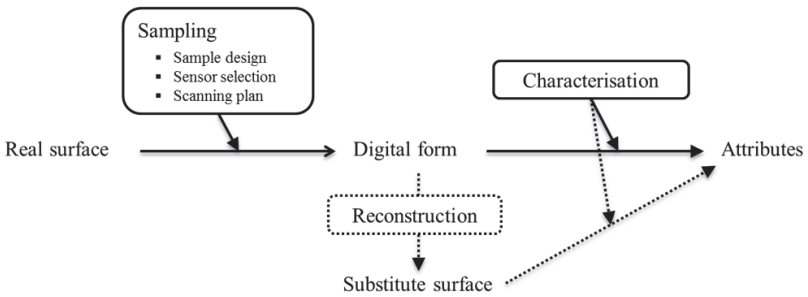

Figure 1. A complete measurement pipeline in modern surface metrology. (solid arrows: the current flow; dashed arrows: the future flow) 


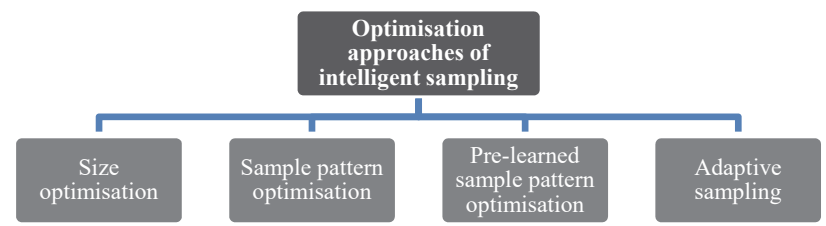

Figure 2. Classification of intelligent sampling methods with respect to optimisation approaches.

Given a specific type of signal and a reconstruction model (e.g. a band-limited signal and sinc reconstruction kernel [8]), the sample size and the positions of sample points can be optimised using intelligent sampling strategies, hence the reconstructed substitute surface can approximate the original surface without, or with limited, error. Many intelligent sampling methods have been proposed recently and these methods can be divided into four categories, as shown in Figure 2, including sample size optimisation, sampling pattern optimisation, pre-learned sampling pattern optimisation and adaptive sampling [12-14]. In the following, a brief review of these solutions are provided. For more details, readers can refer to the latest references in $[6,15,16]$.

\subsection{Brief review of sampling techniques}

Optimisation of the sample size [17-20] or sampling patterns [9, 21, 22] are "blind" methods with very limited intelligence. Such methods assume a stationary or homogeneous distribution of manufacturing errors over a primitive surface model. Hence, an optimised sample design usually has a fixed pattern, such as uniform grids, or Halton or Hamersley patterns. These blind sampling methods generate limited measuring efficiency improvements [13, 23, 24]. Pre-learned sampling optimisation is a relatively new and smart approach which allocates sample points according to a surface complexity analysis of a design model [25-27] or pre-acquired coarse surface data [12-14, 28, 29]. With sufficient a priori knowledge and adequate optimisation function design [12, 14, 27], prelearned intelligent sampling can provide significant efficiency improvements for the measurement of surfaces with spatially heterogeneous characteristic distributions.

In many situations, e.g. in high-accuracy reverse engineering or customised production, a design model or pre-measurement is not feasible, so real-time adaptive sampling is often the most appropriate solution [11]. Adaptive sampling can redirect sampling effort in real time in response to prior observed topography values [13]. Adaptive sampling has been investigated for some time and many methods have been developed [11, 25, 26, 30-41]. Most of the developed methods of adaptive sampling follow a common pipeline, as shown in Figure 3, i.e. iteratively reconstruct previously-acquired data for uncertainty modelling and select next-best-points (NBPs) for adaptive sampling, until a pre-defined stopping criterion is met.

In adaptive sampling, reconstruction of measured surfaces can be conducted in different spaces, e.g. the original object space [25], parametric [40] or an error space [11, 42], which lead to different solutions with different capabilities. An original object space reconstruction allows to directly reconstruct the object surface and this is generally useful for simple 2.5-dimensional surface measurement. Parametric space reconstruction allows to measurement of any complex surfaces with 3-dimensional under-cut features. Error space reconstruction allows to continuously recover the error curve or surface of an object surface with respect to its nominal geometry. Error space reconstruction is especially effective if a normal geometry has been obtained in advance and can provides higher accuracy than general original space reconstruction [11, 42].

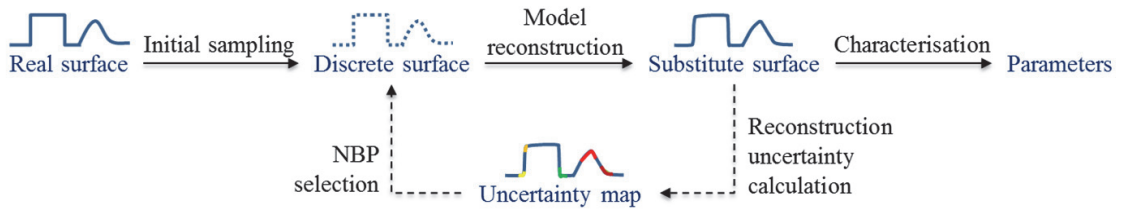

Figure 3. A common adaptive sampling pipeline for surface measurement.

Adaptive sampling can be integrated with blind sample designs for enhanced performance. For example, Hammersley sampling or manufacturing signature pre-learned sampling ${ }_{2}$ can be used as initial sampling strategies for adaptive sampling [43]. In some cases, adaptive sampling uses reconstruction error from a design model for NBP searching [11, 30, 37, 38]. These varied methods have been demonstrated to save sample sizes significantly, e.g. by factors of two to three times [13, 38], with relatively small reconstruction errors.

\subsection{Modelling problem of intelligent sampling}

An essential part of adaptive sampling is that a given method must be able to accurately learn real surface geometry with an accurate mathematical model in a specific space, e.g. the original object [25] space, parametric, or some error spaces [11]. Then, a real surface 
can be approximated by the surrogate model (the real model is usually unknown) without, or with limited, reconstruction error. Recently, a Gaussian process modelling (as a generalised kriging [37, 38])-based intelligent sampling has drawn wide attentions [35, $36,41]$ for their high-degree for modelling flexibility. However, these non-parametric modelling methods are designed for lowdynamic-range (LDR) homogeneous surfaces or near-homogeneous surfaces, e.g. smooth freeform surfaces and low-dynamic-range structured surfaces. Besides, non-parametric modelling currently requires unbearable high computing time costs.

B-spline or NURBS surrogate models defined with several describing parameters [25-27] are widely used to approximate the original design model, with both high accuracy and efficiency. These models can effectively represent simple and complex geometries, e.g. smooth freeform geometry. However, for surfaces with high-dynamic range (HDR) features [44] or highly sparse surfaces with varying local complexities (e.g. local spectral width) at different locations, parametric modelling can easily introduce in large modelling errors due to inaccurate model selections. Figure 4 shows an example of LDR smooth and sharply edged HDR structured surface profiles, where the smooth surface shows homogeneous signal variation everywhere, while the structured surface shows heterogeneous and sparse local bandwidth distributions at different locations.

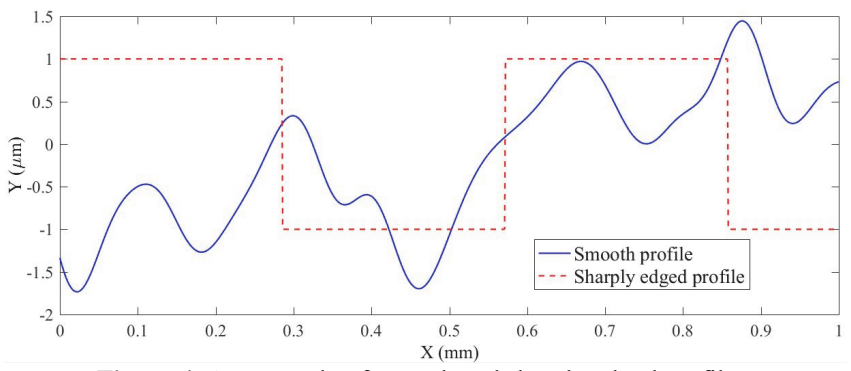

Figure 4. An example of smooth and sharply edged profiles.

To overcome the issue of modelling accuracy and simultaneously take advantage of the efficiency of parametric modelling, a novel adaptive sampling method is proposed in this research based on free-knot B-spline (FKBS) modelling [45-48]. FKBS modelling can automatically select an adequate fitting model and adapt the model hyper-parameters, e.g. knot vectors, to complex surface behaviours. Hence, high reconstruction accuracy from a smart sampling design can be expected. Our experiments show that FKBSmodelling-based adaptive sampling has comparable or higher performance to some state-of-the-art sampling solutions with nonparametric models, e.g. Gaussian process regression or kriging [36, 41], and with less computational cost.

In the following, the proposed solution is described mathematically in part 2 with step-by-step illustrations. Five case studies and discussions are then given in part 3 by conducting the algorithm in different spaces. Part 4 presents conclusions and future work. In this work, only 2D surface profile measurement [49] is considered.

\section{FKBS-based intelligent sampling}

Following with the general pipeline as in Figure 3, the proposed adaptive sampling strategy is carried out in the following loops, including initial sampling, free-knot B-spline modelling, uncertainty estimation, NBP selection and subsequent iterations. For a summary view of the strategy, please forward to section 2.5 .

\subsection{Initial sampling and testing position initialisation}

Initial sampling with a blind strategy can be uniform, Hammersley, Halton pattern or other pre-learned fixed distribution patterns [9, $13,21]$. In this study, a uniform sampling with a random phase shift was applied.

Testing points or candidate points $\left\{x_{m}^{*}\right\}_{m \in M}$ are the positions to be predicted in model reconstruction. In previous research work [11, $36,38]$, a dense and uniform position set with a fixed size is constructed for NBP selection. This solution is not optimal and for large datasets, it is time-consuming as well, especially in areal measurement. In our strategy, a uniform testing point set is initialised first, which is then dynamically refined in adaptive sampling iterations according to local surface complexities.

\subsection{Regularised spline modelling with free knots}

\subsubsection{Regularised B-spline modelling}

B-spline or NURBS fitting has been widely used in surface reconstruction from discrete sample points [50, 51]. For example, given a set of sample positions in $D$-dimensional space $\boldsymbol{x}=\left\{x_{n} \in \mathbb{R}^{D}\right\}_{n \in N}$ and corresponding measurement values $\boldsymbol{y}=\left\{y_{n} \in \mathbb{R}\right\}_{n \in N}$, fitting with B-splines can be expressed with the following 


$$
\boldsymbol{y}=f_{t}(x)=H \alpha+\varepsilon
$$

where $H$ is a $K^{\text {th }}$ order $(K=4$ in this work) B-spline modelling matrix, which relies on a knot position vector $t$ and the sample position vector $\boldsymbol{x}$ and can be easily obtained by using de Boor's algorithm [50], and $\boldsymbol{\varepsilon}$ is the modelling error which follows the independent multivariate normal distribution $\boldsymbol{\varepsilon} \sim \mathbb{N}\left(0, \sigma^{2} \boldsymbol{I}\right)$, with $\boldsymbol{I}$ being the identity matrix. The parameter vector $\boldsymbol{\alpha}$ is usually the only variable of estimation and it can be resolved in a least-squares sense, i.e.

$$
\operatorname{argmin}_{\boldsymbol{\alpha}}\|\boldsymbol{y}-H \boldsymbol{\alpha}\|^{2}: \boldsymbol{\alpha} \in \mathbb{R}^{K}
$$

in which $K$ is usually smaller than $N$ and accounts for the dimension of modelling parameters. Then

$$
\widehat{\boldsymbol{\alpha}}=\left(H^{T} H\right)^{-1} H^{T} \boldsymbol{y} .
$$

The least-squares spline fitting requires the Schoenberg-Whitney-condition on the knot positions [47, 52] to be met so that matrix singularity can be avoided, i.e. the banded matrix $H^{T} H$ is stably invertible. However, this is difficult in practice to satisfy, especially in adaptive sampling with sparse sample distributions, or if a knot vector size is larger than the sample sizes. A regularised minimisation solution with a second order derivative smoothing term is used in this work, which makes the solution $\hat{\alpha}$ stable, i.e.

$$
\operatorname{argmin}_{\boldsymbol{\alpha}}\|\boldsymbol{y}-H \boldsymbol{\alpha}\|^{2}+\mu\left\|f^{r}\right\|_{L_{2}}^{2}
$$

where $\mu$ controls trade-off between the modelling accuracy and smoothness, $\left\|f^{r}\right\|_{L_{2}}$ is the continuous 2-norm of the $r^{\text {th }}$ order $(r=2$ in this work) derivative function, i.e.

$$
\left\|f^{r}\right\|_{L_{2}}^{2}=\int_{X} f^{r}(x)^{2} d x
$$

Because the derivatives of a B-spline are a summation of lower-order B-spline basis functions, the area of a B-spline is proportional to its bounded square box area [53]. Equation (5) can be expressed as

$$
\left\|f^{r}\right\|_{L_{2}}^{2}=\boldsymbol{\alpha}^{r T} S^{T} S \boldsymbol{\alpha}^{r}=\boldsymbol{\alpha}^{T} D^{T} S^{T} S D \boldsymbol{\alpha},
$$

where $D$ is coefficient transfer matrix [50] which realises the following conversion

$$
\alpha_{j}^{r}=(K-r) \frac{\alpha_{j}^{r-1}-\alpha_{j-1}^{r-1}}{t_{j+K-r^{-} t_{j}}} \text {, with } \alpha^{0}=\alpha
$$

and

$$
S=\operatorname{diag}\left(\sqrt{\frac{t_{j+K-r-t_{j}}}{K-r}}\right)
$$

Thus, the curvature minimisation regularised modelling problem above can be solved in following least-squares manner

$$
\widehat{\boldsymbol{\alpha}}=R^{T} H^{T} y, \text { with } R=R^{T}=\left(H^{T} H+\mu D^{T} S^{T} S D\right)^{-1} .
$$

\subsubsection{Free-knot B-spline modelling}

The modelling process above is only applicable to the situation when knot vector $\boldsymbol{t}$ has been defined in advance. In adaptive sampling, with no prior information provided about the surface model, $\boldsymbol{t}$ and $\boldsymbol{\alpha}$ are two variables to be estimated, i.e.

$$
\operatorname{argmin}_{\boldsymbol{t}, \boldsymbol{\alpha}}\left\|\boldsymbol{y}-H_{\boldsymbol{t}} \boldsymbol{\alpha}\right\|^{2}+\mu\left\|f^{r}\right\|_{L_{2}}^{2} \text {. }
$$

Because $\boldsymbol{t}$ and $\boldsymbol{\alpha}$ are linearly separable [53], this free-knot spline modelling problem can be reduced to the optimisation problem of $\boldsymbol{t}$ only by substituting $\boldsymbol{\alpha}$ with equation (9)

$$
\operatorname{argmin}_{\boldsymbol{t}}\left\|\boldsymbol{y}-H_{\boldsymbol{t}} R^{T} H_{\boldsymbol{t}}^{T} \boldsymbol{y}\right\|^{2}
$$

However, equation (11) is a complex non-linear optimisation problem with multiple local minima. Solutions of equation (11) include local Gauss-Newton [47], Gaussian-mixture model optimisation [54], and some global genetic algorithms [55] and simulated annealing algorithms [27]. Global algorithms have been shown to be more accurate than local ones but they cost more computing time. So a compromise solution is used in this work by estimating knots in an approximate but global manner through discrete curvature analysis [45]. This simple knot estimation is found to have high computational efficiency and provide acceptable modelling 
accuracy.

\subsection{Reconstruction uncertainty analysis}

In adaptive sampling, reconstruction uncertainty is usually the only clue for NBP selections. Because B-spline basis functions have a compact support over sub-sampling domains, reconstruction uncertainty usually forms peaks in under-defined areas, i.e. the area far away from existing sample points and with high-density knots placed. Therefore, a well-defined surface reconstruction can be achieved by iteratively sampling at the positions with the maximum uncertainty distributions.

Based on the fitting model in equation (1), prediction of the expected value and uncertainty (square root of variance) at a testing position $x^{*}$ has the following form:

and

$$
\widehat{y}^{*}=\boldsymbol{h}^{T} \widehat{\boldsymbol{\alpha}}=\boldsymbol{h}^{T} R^{T} H^{T} \boldsymbol{y}
$$

$$
U_{y *}=\sqrt{\boldsymbol{h}^{T} R^{T} H^{T} \sum H R \boldsymbol{h}}=\sigma \sqrt{\boldsymbol{h}^{T} R^{T} H^{T} H R \boldsymbol{h}}
$$

where $\boldsymbol{h}$ is the B-spline modelling vector at the new testing position $x^{*}$, and the constant $\sigma$ can normally be pre-set as the measurement uncertainty or estimated with cross-validation [52]. In this research, $\sigma$ is set as $0.1 \%$ of the signal range because an absolute uncertainty magnitude is not our concern (see section 2.4 for more details).

Figure 5a shows the reconstruction uncertainty distribution from twelve uniform sample points for a geometrically heterogeneous surface profile. This heterogeneous surface has higher local complexity in the left area than in the right. Thus, the uniform initial sampling results in the right-half area are well-defined with smaller uncertainties. The left-half area has an uncertainty with several peaks formed at the positions between some neighbouring sample points. By further adaptively allocating thirty-six dense sample points (mainly in the left area), these local uncertainty peaks were reduced in the full sampling domain, as shown in Figure 5b.

It can be observed from equation (13) that the reconstruction uncertainty is independent of sampling values $y$ but dependent on the sampling positions $x$. This does not seem intuitive because any surface will have the same sample design if the B-spline model knots are pre-defined. In fact, equation (13) is provided under the assumption that the model knots are provided with no uncertainty. Therefore, this theoretical uncertainty prediction may underestimate the true uncertainty value [56]. To overcome this problem, an empirical uncertainty estimation, e.g. Jack-knife uncertainty [57] is normally applied

$$
U_{y_{*}}=\sqrt{N-1} \sigma\left(\hat{y}_{n-}^{*}\right)_{n \in N},
$$

where $\sigma$ represents the uncorrected standard deviation and $\hat{y}_{n-}^{*}$ denotes the reconstruction mean value from $N-1$ existing sample points, with the $n^{\text {th }}$ point eliminated. In this work, the Jack-knife uncertainty was used when the design model was unknown. For more information about empirical uncertainty estimation, please refer elsewhere [37, 56].

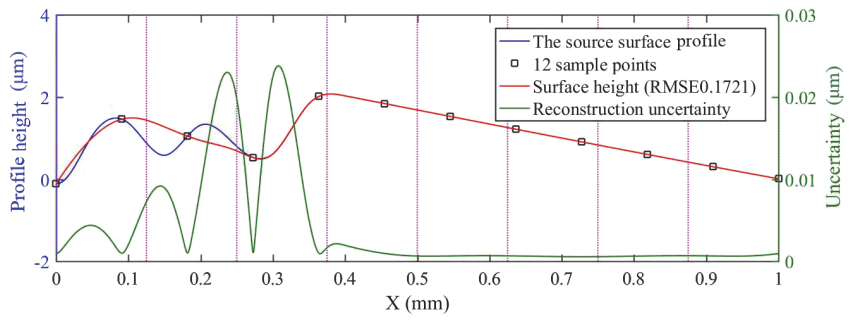

(a) With an initial uniform sampling.

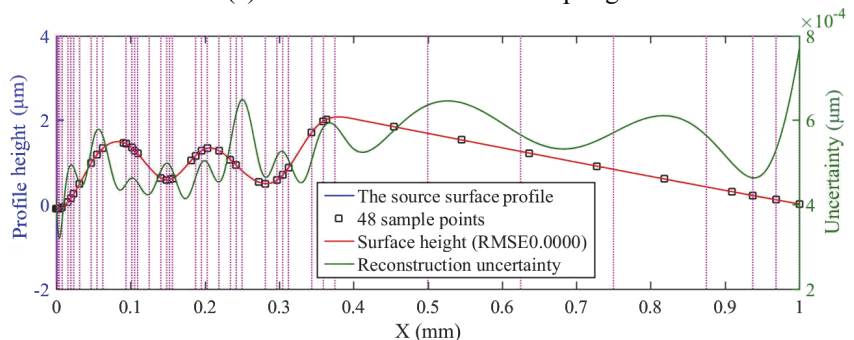

(b) With additional thirty-six adaptive sampling

Figure 5. Update of sample points, testing positions (magenta dashed lines), reconstructed surfaces and uncertainties (note the scale difference) of the adaptive sampling for a fourth order B-spline heterogeneous surface profile with non-uniform interior knots at $[0.05,0.1,0.15,0.2,0.25,0.3,0.35,0.4]$. 


\subsection{Next-best-point (NBP) selection}

Under the framework of greedy algorithms [58], by placing the NBP at the position with the highest uncertainty amplitude, the uncertainty profile or map will be reduced in the next reconstruction iteration. Figure $6 \mathrm{a}-\mathrm{b}$ show that by placing the NBP at $x=0.31$, the local reconstruction uncertainty near to the NBP shrinks to the measuring uncertainty of $0.001 \mu \mathrm{m}$. By further iteratively placing the second and third NBPs, the local reconstruction uncertainties shrink as presented in Figure $6 \mathrm{c}$ and $6 \mathrm{~d}$. Until a total of nine adaptive NBPs are sampled, the reconstruction uncertainties shrink to below the level of measurement uncertainty, as shown in Figure 6e.

NBP selections with the maximum uncertainty or maximum weighted uncertainty have been investigated in earlier research [37, 38], where they were respectively abbreviated as the MaxVar and MaxWVar rules:

$$
\begin{gathered}
\text { MaxVar: } x_{N B P}^{*}=\operatorname{argmax}_{x^{*}} U_{\hat{y}^{*}}^{2} . \\
\text { MaxWVar: } x_{N B P}^{*}=\operatorname{argmax}_{x^{*}} w_{x^{*}} U_{\hat{y} *}^{2} .
\end{gathered}
$$

For the latter rule, a commonly used weight design is the Euclidean distance to the nearest sample, i.e. $w_{x^{*}}=\min _{n \in N} d\left(x^{*}, x_{n}\right)$.

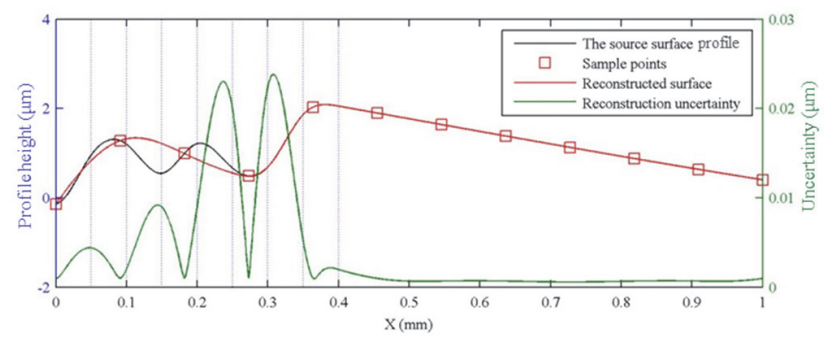

(a)

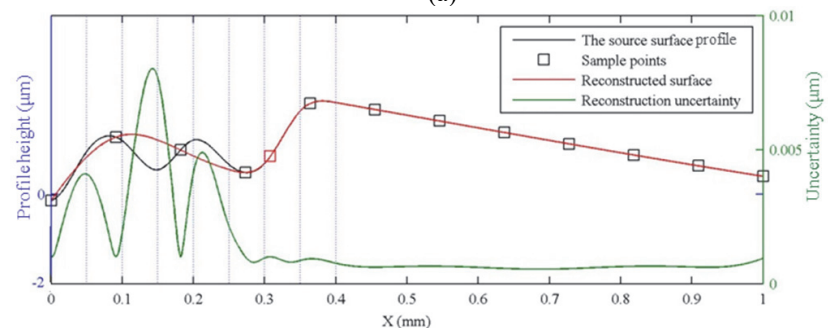

(b)

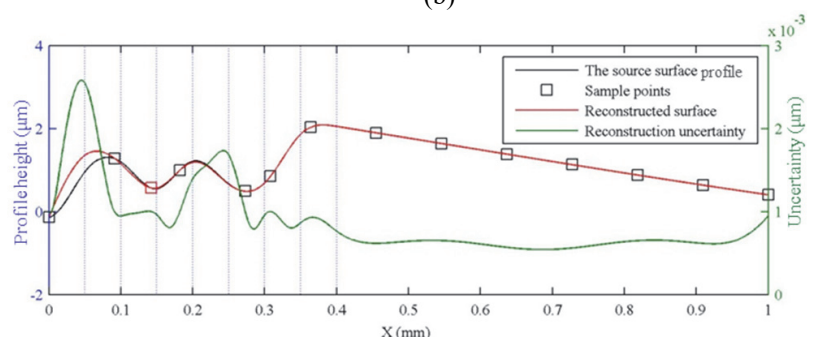

(c)

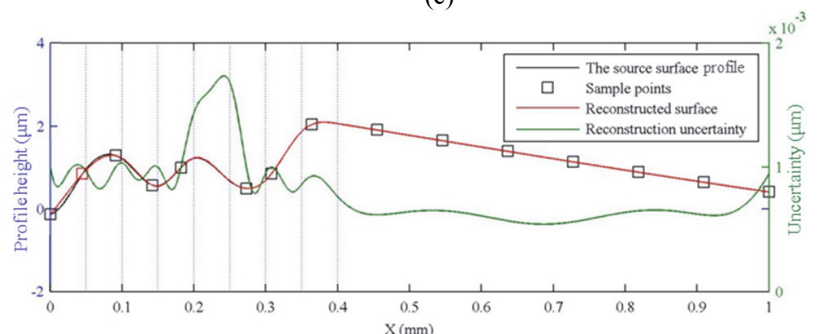

(d) 


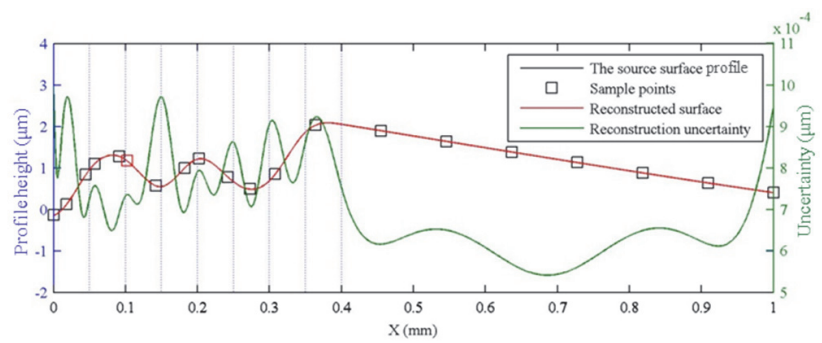

(e)

Figure 6. Reconstruction uncertainty (green, note the scale difference) for initial ten uniform sample points (a) and additional 1(b), 2(c), 3(d) and 9(e) adaptive sample points. (Red squares denote the added sample points in each iteration; Blue dashed lines denote the model knot positions)

If a design model can be provided either by localisation or ordinary fitting, the NBP can be specifically designed at the positions which maximise the peak-to-valley form error, e.g. least-squares or minimum-zone flatness errors. This NBP selection rule was abbreviated as MaxtInc [30, 34, 37, 38], i.e.

$$
\text { MaxtInc: } x_{N B P}^{*}=\operatorname{argmax}_{x^{*}}\left[e\left(x, y, x^{*}, y^{*}\right)-e(x, y)\right] \text {. }
$$

If equation (17) leads to no NBP selection during a sampling iteration, a switch rule can then be applied by switching the MaxtInc rule to MaxVar or MaxWVar rule.

Previous research have demonstrated that the MaxtInc to MaxWVar switch rule is especially effective for peak-to-valley form error measurement, e.g. for least-squares or minimum-zone straightness error measurement. For general geometry measurement, e.g. to minimise a root-mean-squared measurement error, MaxVar and MaxWVar rules are preferred [37, 38]. In this research, MaxWVar is used as the default NBP selection rule in this research if not specially specified.

\subsection{Adaptive iterations and strategy summary}

With the the above processing loops ready, the existing adaptive sample set $\left\{x_{n}, y_{n}\right\}_{n \in N}$ can be updated with $N=N+1$. Then the testing position set $\left\{x_{m}^{*}\right\}_{m \in M}$ is also refined by inserting a new testing point in the centre of previously divided spaces, which indicates the total number of testing points $M$ is augmented by one over each iteration. With such adaptive binary-tree (or quad-tree for 2D sampling [59]) space partitioning, a sampling space can be hierarchically organised and adaptively refined.

For example, a whole sampling space is set as the root node and has two children nodes, one is the left half space and the other one is the right half space. Also, each node space can recursively have its own refined subspace children. Figure 5a shows a uniform space partitioning with eight leaf nodes. When adaptive sampling starts, one of the leaf subspaces to which a NBP belongs is refined by two children leaf subspaces. Then, the total number of testing points increases by one after each iteration. Figure $5 \mathrm{~b}$ shows an adaptive space partitioning result after thirty-six adaptive iterations, in which the sample points are selected as the centres of the adaptively refined subspaces.

Here, the proposed FKBS modelling-based adaptive sampling is summarised as follows:

1) Initial sampling with a blind strategy and initialising testing positions with a spatial partitioning tree;

2) FKBS modelling with the existing sample points $\left\{x_{n}, y_{n}\right\}_{n \in N}$ by estimating model parameters $\hat{\theta}_{N}=\{\hat{\alpha}, \hat{t}\}$;

3) Predicting uncertainty at the existing testing positions $\left\{x_{m}^{*}\right\}_{m \in M}$;

4) Selecting the NBP $x^{*}$ selection and acquiring the sample value $y^{*}$;

5) Updating the sample points $\left\{x_{n}, y_{n}\right\}_{n \in N}$ and refining the testing position $\left\{x_{m}^{*}\right\}_{m \in M}$ with $N=N+1, M=M+1$;

6) Repeat steps 2-5 until a stopping criterion is achieved.

Within the iterative strategy above, stopping criteria are a critical factor to the performance of adaptive sampling. Some commonly applicable criteria have been used in previous research, such as the predefined sample size [37, 38], predefined maximum reconstruction error $[11,30,37,60]$ and uncertainty [35-38]. With combined use of these stopping criteria, adaptive sampling can be effectively applied. In this work, sample size was used as the only stopping criterion to validate the performance of the proposed sampling strategy, by comparing with control group methods.

\section{Experiments and discussions \\ 3.1 Preparation of specimen and sampling conditions}


A study of the proposed adaptive sampling strategy step by step on a heterogeneous surface profile has been given in section 2 , in which case the surface model parameter $\boldsymbol{t}$ was pre-defined and only the theoretical uncertainty was used. In this section, the proposed sampling strategy is tested using an empirical uncertainty analysis by not providing any prior information.

Engineered surfaces can be generally classified into random and deterministic surfaces [1]. The former can be widely found in the natural surface texture of general machining processes, which usually have homogeneous behaviour. Uniform or blind sampling strategies $[8,9]$ work effectively for this type of surface. Deterministic surfaces have designed shapes such as MEMS and diverse structured or freeform surfaces. These advanced surfaces normally have sharply edged features or HDR properties [1, 44].

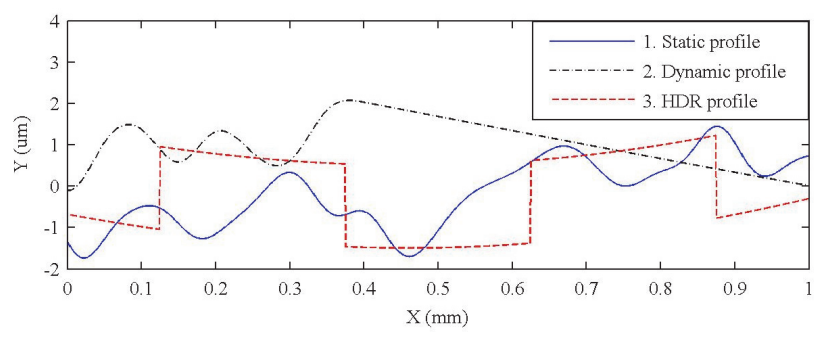

(a)
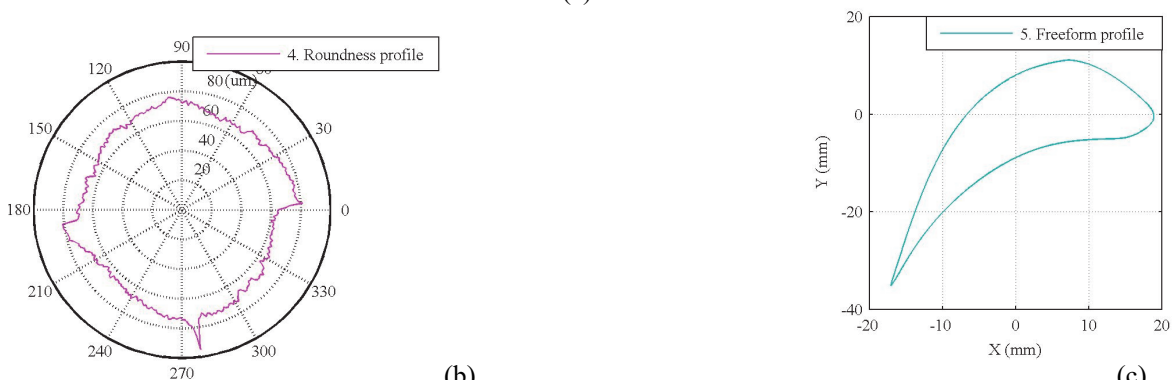

(b)

(c)

Figure 7. Five typical surface profiles to be tested.

In this experiment, a range of surface examples, including three simulated profiles with respectively smooth, heterogeneously smooth and sharply edged geometries, a measured roundness profile and a measured sharp-edge enclosed freeform profile (see Figure 7) are chosen for verification. The simulated smooth profile is a randomly generated uniform knot spline space curve. The simulated heterogeneously smooth profile is the one analysed in section 2 . The simulated sharply edged profile is a composition of a step signal and parabolic base form. The roundness profile data in Figure $7 \mathrm{~b}$ comes from reference [11], which has also been investigated by other researchers for peak-to-valley form error evaluation [37, 38]. The sharp-edge enclosed freeform profile, shown in Figure 7c, comes from an aero-engine turbine blade and was measured with laser triangulation [51, 61].

Sampling experiments with different strategies and sample sizes were then applied in which uniform, jittered uniform (JU) [13] and the recently highlighted kriging modelling-based adaptive sampling [36-38] were used as the references. Sample sizes varied from $14,27,54,100$ until 200 among which, the size 27 was specially tested to compare with the method in reference [11], while other sample points were compared with the other studies as in references [37,60]. The default NBP selection rule used in this experiments is MaxWVar.

For sampling performance verification, root-mean-squared (RMS) and peak-to-valley (PV) reconstruction errors (RMSE and PVE) [13] are used as the performance metrics, i.e.

$$
\begin{gathered}
\varepsilon_{\mathrm{RMS}}=\sum\left(\hat{y}_{n}-\bar{y}_{n}\right)^{2} / N, \text { with } n \in N, \\
\varepsilon_{\mathrm{pv}}=P_{z}\left(\hat{y}_{n}\right),
\end{gathered}
$$

where $\hat{y}$ and $\bar{y}$ are respectively the reconstruction model and the design model in a specific space and $P_{z}$ represents the extreme height difference between the highest peak and the lowest valley [4]. The RMS error is analysed for the first three cases and the last freeform case, while the PV error is analysed for the fourth roundness case to compare with the results in reference [11]. Normalised RMSE and PV errors are also used in the statistical analysis for a unified performance comparison between different cases, i.e.

$$
\begin{gathered}
\varepsilon_{\mathrm{RMS}} \%=\frac{\varepsilon_{\mathrm{RMS}}}{P_{z}\left(\bar{y}_{n}\right)} \times 100 \%, \text { with } n \in N, \\
\varepsilon_{\mathrm{pv} \%}=\frac{\left|P_{z}\left(\hat{y}_{n}\right)-P_{z}\left(\bar{y}_{n}\right)\right|}{P_{z}\left(\bar{y}_{n}\right)} \times 100 \% \text {, with } n \in N .
\end{gathered}
$$




\subsection{Typical sampling results \\ 3.2.1 Smooth surface profile}

Some typical sampling results are presented here. For the smooth profile from a uniform knot spline space, the developed FKBS adaptive sampling presented unstable results when 27 sample points (SPs) were applied, but quickly converged to a stable reconstruction curve when 54 or more SPs were used. In Figure 8, a 27 SP-based sampling-reconstruction curve has an RMS error of $0.128 \mu \mathrm{m}$. When the sample size was doubled to 54 , the sampling reconstruction RMSE quickly degenerated to only $5 \%$ of the previous value, i.e. $0.002 \mu \mathrm{m}$.

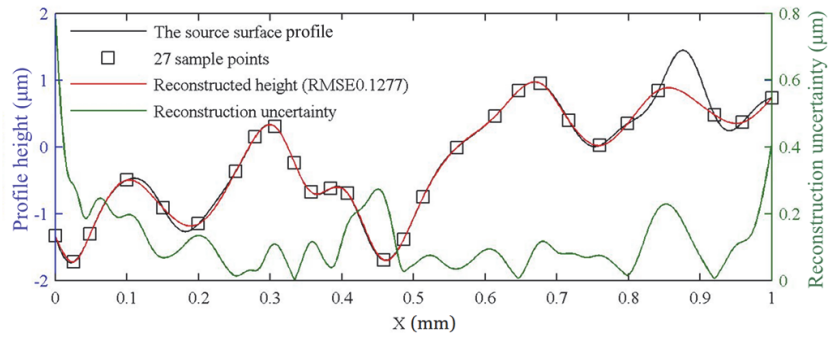

(a) with $27 \mathrm{SPs}$

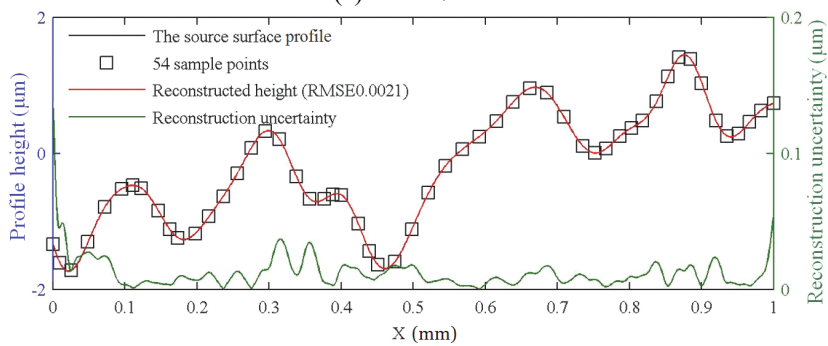

(b) with $54 \mathrm{SPs}$

Figure 8. Typical FKBS adaptive sampling designs for the smooth profile.

Because the selected smooth profile has homogeneous surface complexities over the abscissa, the resulting adaptive sample points exhibited a near-uniform distribution. The reconstruction errors of the control groups are listed in Table 1, from which it can be found that the performance of the FKBS adaptive sampling is similar to that of the uniform methods with sample size 27 but outperforms the conventional methods with sample size 54. However, the FKBS sampling performed no better than the kriging-based adaptive method for this smooth surface.

\begin{tabular}{ccccc}
\hline Sample sizes & Uniform & Jittered uniform & Kriging Adaptive & 0.034 \\
\hline $\mathbf{2 7}$ & 0.080 & 0.129 & 0.127 \\
$\mathbf{5 4}$ & 0.021 & 0.029 & 0.001 & 0.002 \\
\hline
\end{tabular}

Table 1. RMSEs of different sampling methods on the smooth profile in micrometres.

\subsubsection{Heterogeneously smooth surface profile}

A typical sampling design of the proposed FKBS adaptive sampling for the heterogeneous profile is plotted in Figure 9. Because this heterogeneous profile has higher local complexity in the left area, the FKBS sampling method intelligently found this under-defined area and allocated denser sample points there. With the sample size changing from 27 to 54, the FKBS sampling provided a significant accuracy improvement by reducing the reconstruction RMSE to $10 \%$ of the previous value. Simultaneously, the blind uniform methods reduced the reconstruction errors to about one-fourth of the previous values (see Table 2). From Table 2, it can be seen that the proposed FKBS sampling method outperformed all other tested methods in sample size 27 and 54, including the state-of-the-art kriging-based adaptive sampling [35-38]. 


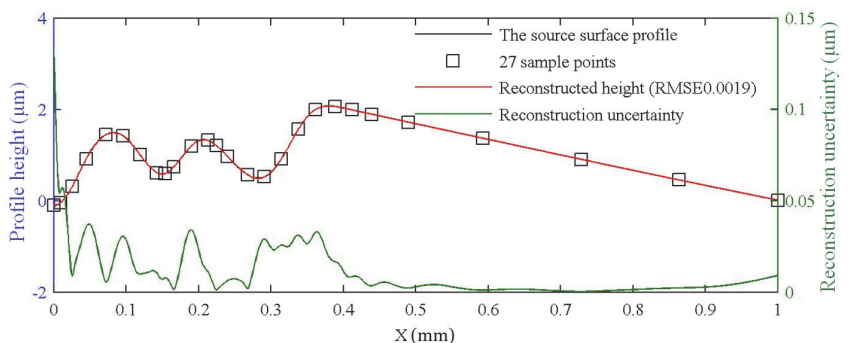

(a) with $27 \mathrm{SPs}$

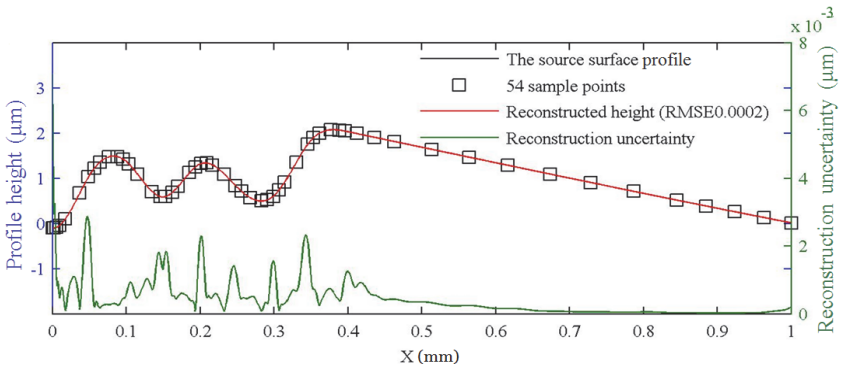

(b) with $54 \mathrm{SPs}$

Figure 9. Typical FKBS adaptive sampling designs for the heterogeneous profile.

\begin{tabular}{ccccc}
\hline $\begin{array}{c}\text { Sample } \\
\text { sizes }\end{array}$ & Uniform & $\begin{array}{c}\text { Jittered } \\
\text { uniform }\end{array}$ & $\begin{array}{c}\text { Kriging } \\
\text { Adaptive }\end{array}$ & $\begin{array}{c}\text { FKBS } \\
\text { Adaptive }\end{array}$ \\
\hline $\mathbf{2 7}$ & 0.054 & 0.088 & 0.010 & 0.002 \\
$\mathbf{5 4}$ & 0.015 & 0.018 & 0.001 & 0.0002 \\
\hline
\end{tabular}

Table 2. RMSEs of different sampling methods for the heterogeneous profile in micrometres.

\subsubsection{Structured surface profile with sharp edges}

The most difficult case for intelligent sampling design is a structured surface with sharp edges. The simulated structured surface profile in this study has smooth and stationary behaviour over the sampling domain but with four break points at $[0.125,0.375,0.625$, 0.875]. These break points have high local slope and curvatures. Most current sampling strategies failed to deal with these step-like surfaces, though some artificial neural network models [62] are in development for this highly nonlinear problem.

Typical sampling designs and reconstruction curves are presented in Figure 10, with the FKBS adaptive sampling strategy. The FKBS method presented favourable adapting behaviour across the high local spatial frequency areas by allocating dense sample points around the break point positions and reconstructing with limited oscillation. With other intelligent strategies, such as the kriging method, a 54 SPs design is presented in Figure 11a. Although the kriging adaptive method [35-38] also allocated dense SPs around the break points, the reconstruction showed prediction bias to a zero-mean line at positions with sparse SPs. Adaptive sampling in [11] is another spline modelling-based method with spline knots equal to sample positions, hence sample points were exactly interpolated. Unfortunately, this method [8] produced obvious oscillations around the break points (see Figure 11b).

Table 3 summarises the performance of the proposed method with the control groups. It can be observed that the conventional uniform methods presented little accuracy improvement by increasing SPs from 27 to 54. Simultaneously, the kriging-based method presented a small improvement. The FKBS method demonstrated an evident accuracy improvement by reducing the reconstruction error to one-fourth of the previous value.

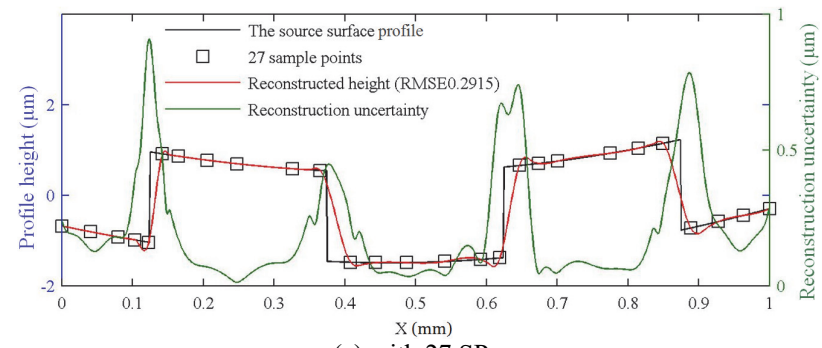

(a) with $27 \mathrm{SPs}$ 


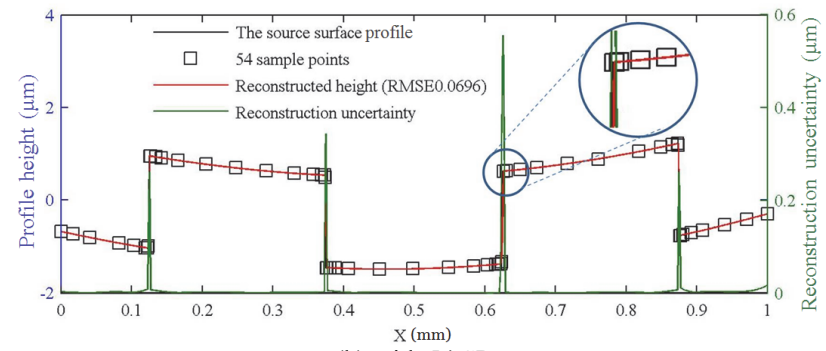

(b) with 54 SPs

Figure 10. Typical FKBS adaptive sampling designs for the shapely edged profile.

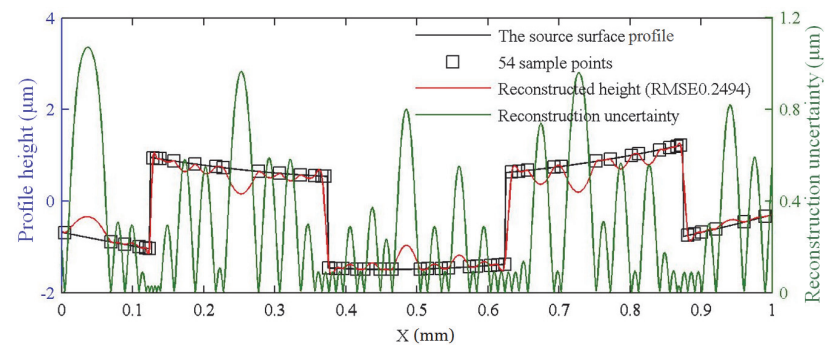

(a) with kriging-based adaptive sampling

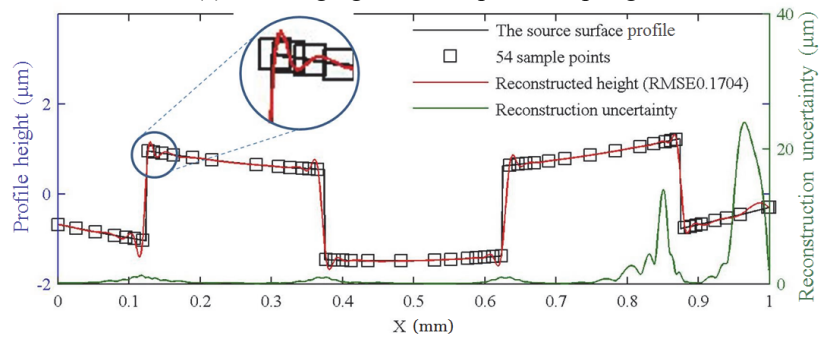

(b) with adaptive sampling from [8]

\subsubsection{Real roundness surface profile}

The roundness surface profile comes from a machined cylindrical shaft which involves a composition of large-scale form error and small-scale texture. The texture amplitude is about one-tenth of the form error and presents a homogeneous distribution over the sampling domain. However, the large-scale form has some sharp changes at four rotational positions (see Figure 7b). Previous research [37, 38] focused on the inspection of the PV form error in which kriging adaptive methods were demonstrated to significantly save the sample size by up to $50 \%$ with the MaxtInc-MaxWVar NBP selection rule, compared to the method in reference $[8]$.

\begin{tabular}{ccccccc}
\hline Sample & Uniform & Jittered & \multicolumn{2}{c}{ Kriging adaptive } & \multicolumn{2}{c}{ FKBS Adaptive } \\
\cline { 4 - 7 } sizes & & uniform & MaxWVar & Switch & MaxWVar & Switch \\
\hline $\mathbf{2 7}$ & $53.4 \%$ & $41.6 \%$ & $44.3 \%$ & $30.7 \%$ & $42.2 \%$ & $15.0 \%$ \\
$\mathbf{5 4}$ & $38.7 \%$ & $46.3 \%$ & $21.6 \%$ & $27.1 \%$ & $19.8 \%$ & $11.9 \%$ \\
\hline
\end{tabular}

Table 4. Normalised PVEs of different sampling methods on the roundness case.

\begin{tabular}{ccccccc}
\hline Sample sizes & \multirow{2}{*}{ Uniform } & \multirow{2}{*}{ Jittered uniform } & \multicolumn{2}{c}{ Kriging Adaptive } & \multicolumn{2}{c}{ FKBS Adaptive } \\
\cline { 3 - 6 } & & & MaxWVar & Switch & MaxWVar & Switch \\
\hline $\mathbf{2 7}$ & 2.285 & 2.042 & 2.79 & 2.877 & 2.51 & 2.137 \\
$\mathbf{5 4}$ & 2.003 & 1.715 & 1.89 & 2.193 & 1.51 & 1.462 \\
\hline
\end{tabular}




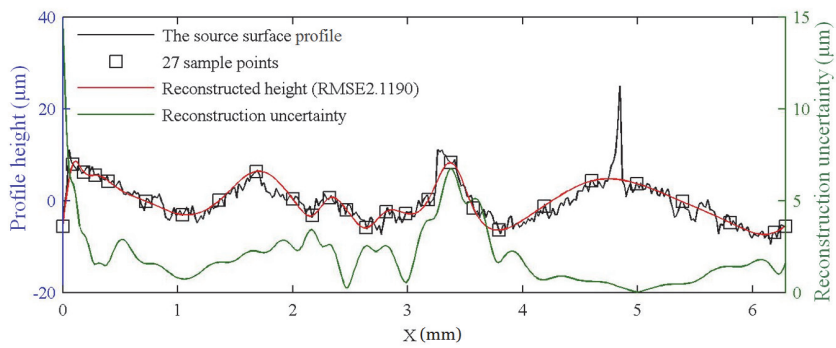

(a) with $27 \mathrm{SPs}$

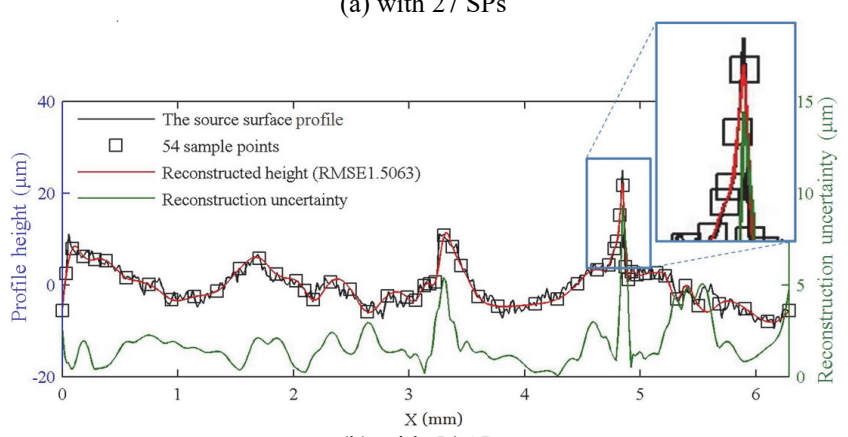

(b) with 54 SPs

Figure 12.Typical FKBS adaptive sampling designs for the roundness profile.

With the MaxtInc-MaxWVar switch NBP selection rule for both kriging and FKBS adaptive sampling, a PVE evaluation test was carried out and the results are shown in Table 4. The corresponding RMSEs of each test are listed in Table 5. A typical sampling reconstruction result of the FKBS adaptive method under the switch rule is also presented in Figure 12, in which it can be found that the proposed method can stably find the highest surface peak.

In this presented case, the FKBS sampling showed higher PVE evaluation accuracy than the kriging and conventional uniform methods, but exhibited no significantly higher performance for RMSE evaluation. One reason behind this could be the high amplitude texture, which results to over-fitting with the proposed FKBS reconstruction method. It was also found that the adaptive sampling strategies are sensitive to the initial sampling conditions. Therefore, a statistical analysis needs to be carried out which is presented in section 3.3 .

\subsubsection{Real freeform surface profile}

The freeform surface profile has re-entrant 2D geometry. Its coordinate point cloud needs to be mapped to a parametric space [63], hence the profile can be described as a parametric spline part plus a single-value error function part, i.e.

$$
\boldsymbol{p} \rightarrow r: \boldsymbol{p}=\boldsymbol{p}(u)+r(u) \boldsymbol{n}(u),
$$

where $\boldsymbol{p}(u)$ is an approximated spline model by extending equation (1) to a $2 \mathrm{D}$ space, i.e. $\boldsymbol{p}(u)=[x(u), y(u)]^{T}=$ $\left[\boldsymbol{h}_{x}(u), \boldsymbol{h}_{y}(u)\right]^{T} \boldsymbol{\alpha}, \boldsymbol{n}(u)$ is the normalised normal vector, and $r(u)$ is the separated residual error curve for adaptive sampling. In other words, the sampling experiment was carried out in a parametric space.

In this study, the approximated parametric model was manually constructed with a NURBS CAD toolbox. Then the residuals $r(u)$ of the measured profile were extracted as the black curve in Figure 13, in which the abscissa has been normalised. This real freeform profile has smooth form geometry over the majority of the sampling space but a local peak around the leading edge. With the proposed adaptive sampling, the freeform profile was reconstructed with limited reconstruction error (see Table 6). In particular, the proposed method could stably find the small peak as shown in Figure $13 \mathrm{~b}$ near the leading edge with 54 sample points, by using the MaxtIncMaxWVar switch NBP selection rule. 


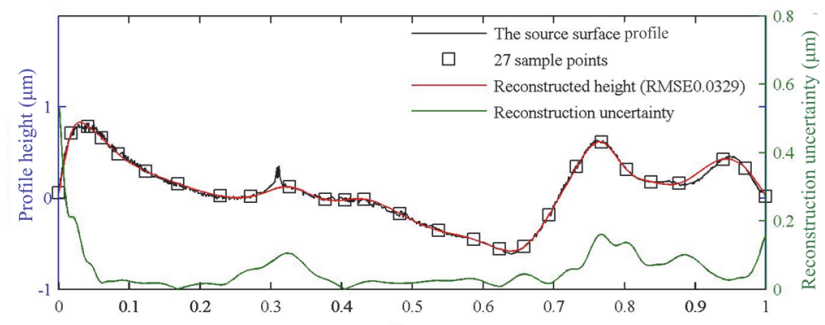

(a) with 27 SPs (MaxWVar)

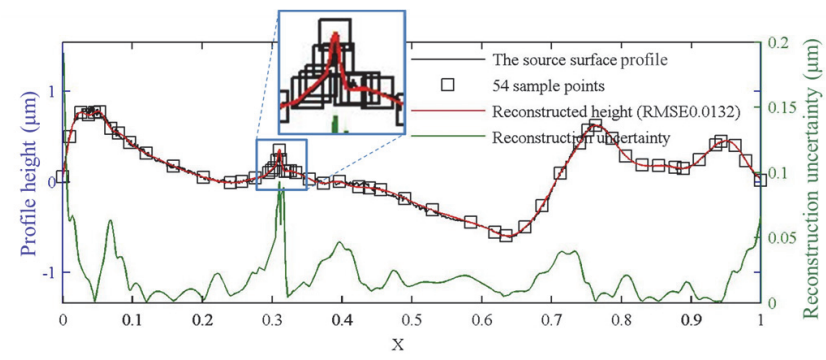

(b) with 54 SPs (MaxtInc-MaxWVar switch)

Figure 13. Typical FKBS adaptive sampling designs for the parameterised freeform profile.

\begin{tabular}{ccccc}
\hline Sample sizes & Uniform & Jittered uniform & Kriging Adaptive & FKBS Adaptive \\
\hline $\mathbf{2 7}$ & 0.033 & 0.037 & 0.044 & 0.033 \\
$\mathbf{5 4}$ & 0.021 & 0.018 & 0.024 & 0.013 \\
\hline
\end{tabular}

Table 6. RMSEs of different sampling methods on the freeform case in micrometres.

In Figure 14, we plot a reconstruction of the sampling results back to the original object space in which, the proposed adaptive sampling showed more exact reconstruction performance at the edge position of the turbine blade.

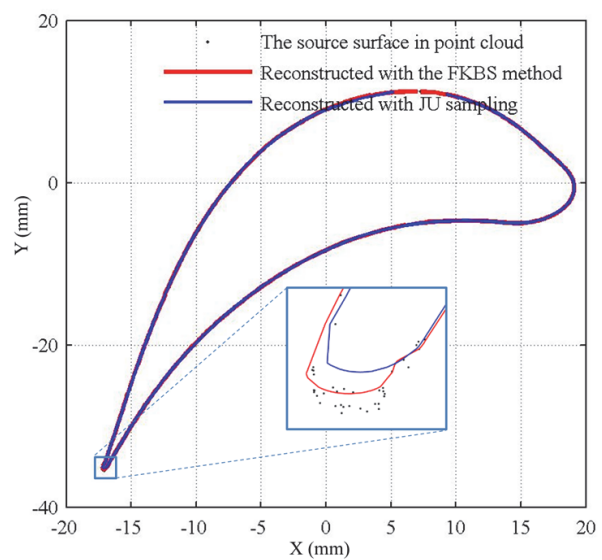

Figure 14. Reconstruction of the source freeform profile from the parametric space back to the original object space.

\subsection{Statistical verification}

\subsubsection{Sampling accuracy}

Adaptive sampling is widely known to be sensitive to initial conditions, e.g. the initial sample size and positions. A statistical verification of sampling performance is normally necessary. In this study, an initial sampling was conducted by randomly collecting five jittered uniform sample points from each dense data. Then, twenty repetitive experiments were run for each method and each sample size. To achieve the comparability among different cases, normalised RMS errors in equation (20), or PV errors for the roundness case in equation (21), were analysed.

In Figure 15, the statistical test results on the five typical surface examples are presented. It can be observed that the proposed FKBSbased adaptive sampling has higher flexibility and reconstruction accuracy for different types of ideal surfaces for the first three cases. For the last two real surface cases, the proposed sampling also exhibits similar or better performance in the PV or RMS form error comparison. 


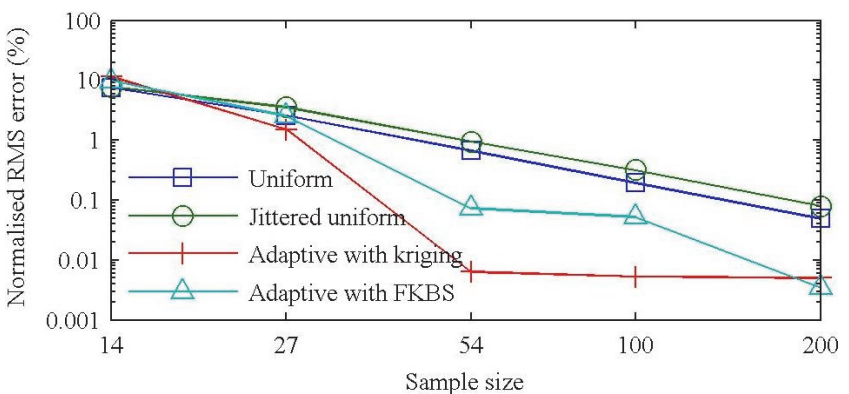

(a) For the smooth profile

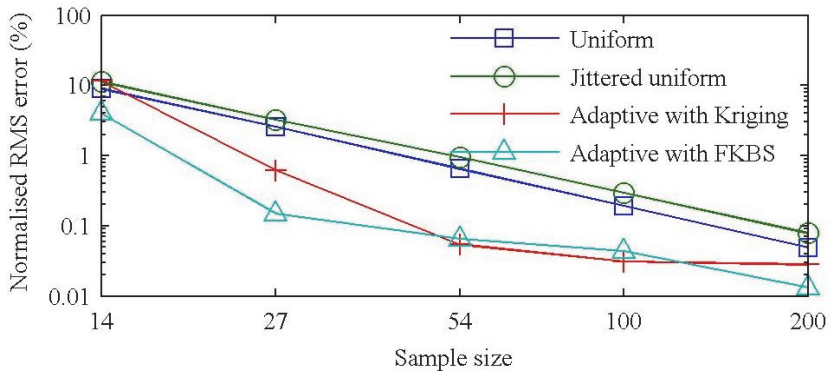

(b) For the heterogeneously smooth profile

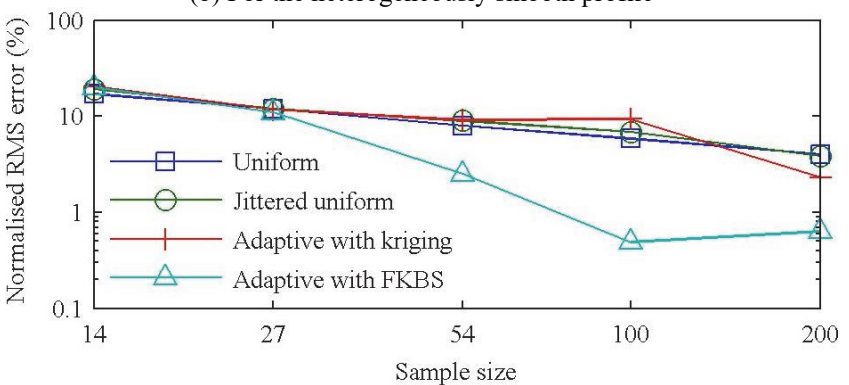

(c) For the sharp-edge structured profile

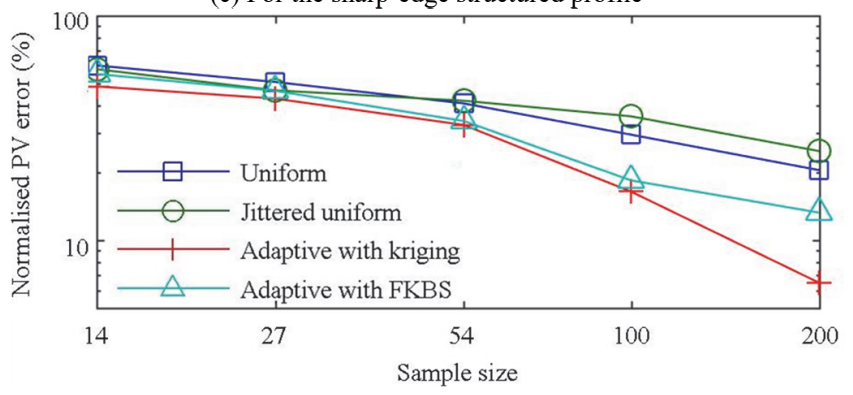

(d) For the real roundness profile

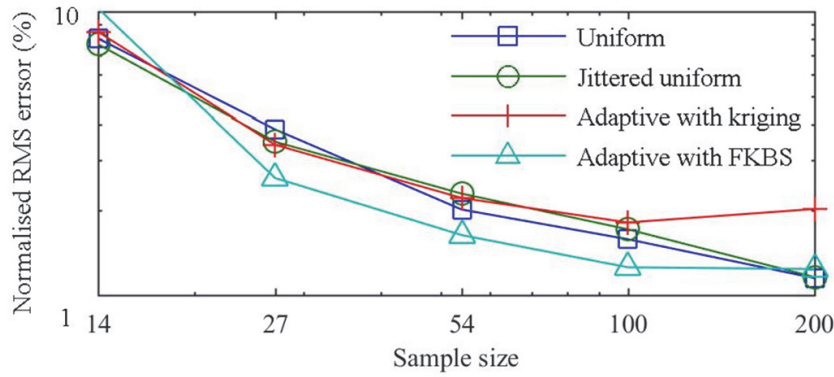

(e) For the real freeform profile

Figure 15. Statistics of the reconstruction errors for the measurement of different specimen with different sampling strategies and sizes ( $y$-axis in log-scale)

It should be noted that when noise is present in the real cases, the proposed sampling and corresponding reconstruction method may over-fit the noise when the sample size increased to two-hundred (see Figure 15e). In this situation, conventional uniform sampling methods performed better than the proposed method and the kriging-based adaptive sampling methods. Figure 16a presents such an 
unexpected example of over-fitting, in which the reconstruction curve passes through the sample points but oscillations are also produced. To avoid over-fitting, a simple solution is to either use a small number of sample points or provide a larger number of knot points in the modelling process. Figure $16 \mathrm{~b}$ presents a good example with no over-fitting and oscillation by pre-setting the number of knots to two hundred.

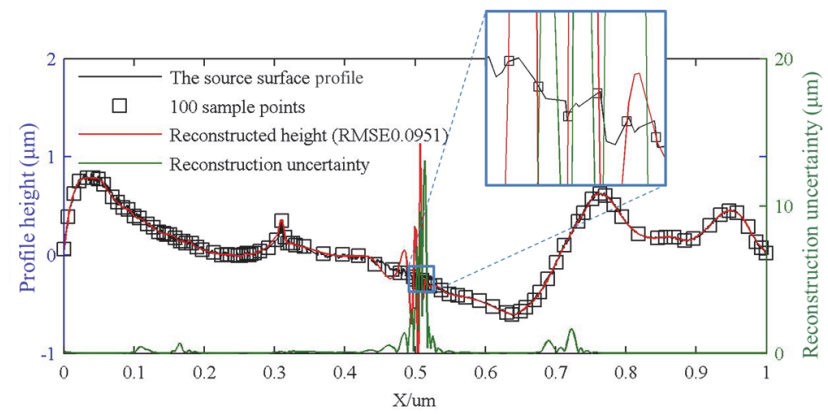

(a) with 100 knot points

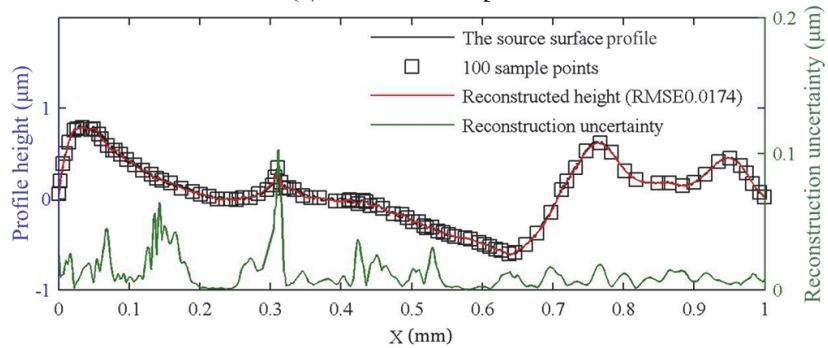

(b) with 200 knot points

Figure 16. An example of over-fitting with the proposed sampling and reconstruction methods with one-hundred sample points.

\subsubsection{Computing time}

The recently highlighted kriging-based adaptive sampling has shown good performance in terms of reducing the number of sample points. However, a barrier exists in practical application of this method as it is computationally expensive [38]. In contrast, during the five typical sampling test cases above, the proposed spline regression-based sampling method showed significant computing time reduction of at least one order of magnitude compared to that from the kriging-based methods. A statistic of the computing time is listed in Figure 17. These experimental data are obtained using MATLAB in a normal computer with 2.4GHz Intel I5 CPU.

When the sample size was pre-set to two hundred, the kriging-based method takes about $100 \mathrm{~s}$ for a full test. In contrast, the proposed method takes about $200 \mathrm{~ms}$. This indicates that the proposed method can be efficiently applied in practical measurement with limited delay between two successive sampling actions.

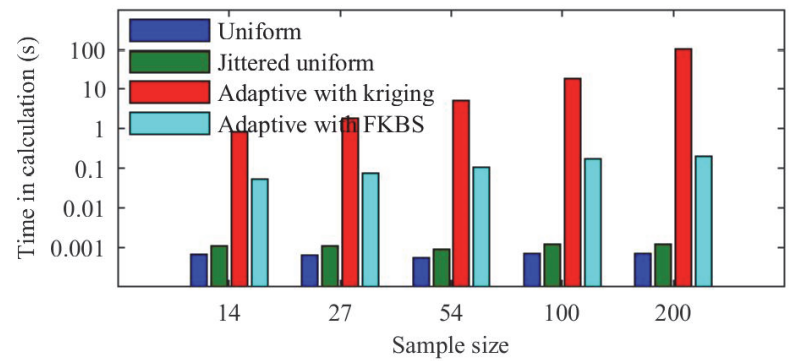

Figure 17. The computation time spent under different sampling conditions (ordinates in log-scale).

\section{Conclusions and future work}

In this research, an uncertainty analysis-guided adaptive sampling method based on free-knot B-spline modelling is proposed. This proposed method has been tested on three simulated surfaces and two measured surface profiles. By comparing with recently krigingbased adaptive sampling and conventional uniform methods, FKBS modelling-based adaptive sampling show similar or better performance for general low-dynamic-range smooth surface profile measurement (see case studies 1,2 and 4 ). For high-dynamic- 
range surface profiles with sharp edges (see case studies 3 and 5), FKBS strategies can significantly outperform conventional uniform methods and the recently popularised kriging-based methods.

Regarding to computational cost, the proposed adaptive sampling shows significant computing time reduction from that of krigingbased methods. Our typical computing time in $200 \mathrm{~ms}$ with MATLAB can be well expected in practical measurement applications.

Intelligent sampling research with parametric models for high-dynamic-range or sparse surfaces is still in its early stage of development. A very important future work is to extend the 2D sampling method to $3 \mathrm{D}$ cases for areal surface measurement. In this case, the computational complexity may increase in a squared sense with tensor-product methods and unpredictable behaviour may be caused by inconsistent 2D border regions. Therefore, we need advanced 2D spline models with sparse structure support for flexible surface modelling. Some typical sparse 2D B-spline models include multi-level B-splines, T-splines and a recently-developed locallyrefined B-splines [59]. With these advanced mathematical models, we can expect the advent of 3D surface measurement-oriented adaptive sampling algorithms in soon, with both small computing cost and high modelling flexibility for high-dynamic-range and sparse surfaces.

\section{Acknowledgement}

This project is supported by National Natural Science Foundation of China (Grant No. 51705178 and No. 51475190) and the Key Project of Natural Science Foundation of Hubei Province of PR China (Grant No. 2017AAA001).

\section{References}

[1] X. J. Jiang and D. J. Whitehouse, "Technological shifts in surface metrology," CIRP Annals - Manufacturing Technology, vol. 61, no. 2, pp. 815-836, // 2012.

[2] R. Leach, Fundamental Principles of Engineering Nanometrology. Elsevier Science, 2014.

[3] B. Adcock, A. Hansen, B. Roman, and G. Teschke, "Chapter Four - Generalized Sampling: Stable Reconstructions, Inverse Problems and Compressed Sensing over the Continuum," in Advances in Imaging and Electron Physics, vol. 182, P. W. Hawkes, Ed.: Elsevier, 2014, pp. 187-279.

[4] ISO 25178-2: 2012, "Geometrical product specification (GPS) - Surface texture: areal - part 2: terms, definitions and surface texture parameters," International Organization of Standardization.

[5] ISO 17450-1:2011, "Geometrical product specifications (GPS) -- General concepts -- Part 1: Model for geometrical specification and verification," 2011.

[6] G. Moroni and S. Petrò, "Geometric Inspection Planning as a Key Element in Industry 4.0," in Proceedings of 3rd International Conference on the Industry 4.0 Model for Advanced Manufacturing, Cham, 2018, pp. 293-310: Springer International Publishing.

[7] C. E. Shannon, "Communication In The Presence Of Noise," Proceedings of the IEEE, vol. 86, no. 2, pp. 447-457, 1998

[8] A. Aldroubi and K. Grochenig, "Nonuniform Sampling and Reconstruction in Shift-Invariant Spaces," SIAM Rev., vol. 43, no. 4, pp. 585-620, 2001

[9] T. C. Woo and R. Liang, "Dimensional measurement of surfaces and their sampling," Computer-Aided Design, vol. 25, no. 4, pp. $233-239,4 / / 1993$.

[10] A. Weckenmann, H. Eitzert, M. Garmer, and H. Weber, "Functionality-oriented evaluation and sampling strategy in coordinate metrology," Precision Engineering, vol. 17, no. 4, pp. 244-252, 1995.

[11] R. Edgeworth and R. G. Wilhelm, "Adaptive sampling for coordinate metrology," Precision Engineering, vol. 23, no. 3, pp. 144-154, 1999.

[12] B. M. Colosimo, G. Moroni, and S. Petrò, "A tolerance interval based criterion for optimizing discrete point sampling strategies," Precision Engineering, vol. 34, no. 4, pp. 745-754, 2010.

[13] J. Wang, X. Jiang, L. A. Blunt, R. K. Leach, and P. J. Scott, "Intelligent sampling for the measurement of structured surfaces," Measurement Science and Technology, vol. 23, no. 8, p. 085006, 2012.

[14] G. Moroni and S. Petrò, "Optimal inspection strategy planning for geometric tolerance verification," Precision Engineering, vol. 38, no. 1, pp. 71-81, 2014/01/01/ 2014.

[15] G. Moroni and S. Petrò, "Coordinate Measuring Machine Measurement Planning," in Geometric Tolerances: Impact on Product Design, Quality Inspection and Statistical Process Monitoring, B. M. Colosimo and N. Senin, Eds. London: Springer London, 2011, pp. 111-158.

[16] E. Morse et al., "Tolerancing: managing uncertainty from conceptual design to final product," CIRP

Annals, vol. 68, no. 2, p. In print, 2018.

[17] H. T. Yau and C. H. Menq, "A unified least-squares approach to the evaluation of geometric errors using discrete measurement data," International Journal of Machine Tools \& Manufacture, vol. 36, no. 11, pp. 1269-1290, 1996.

[18] W. P. Dong, E. Mainsah, and K. J. Stoutt, "Determination of appropriate sampling conditions for three-dimensional microtopography measurement," International Journal of Machine Tools and Manufacture, vol. 36, no. 12, pp. 1347-1362, 1996

[19] R. J. Hocken, J. Raja, and U. Babu, "Sampling Issues In Coordinate Metrology," Manuf Rev, vol. 6, pp. 282-294, 1993.

[20] V. N. N. Namboothiri, "On determination of sample size in form error evaluation using coordinate metrology," International Journal of Production Research, vol. 37, no. 4, pp. 793-804, 1999.

[21] G. Lee, J. Mou, and Y. Shen, "Sampling strategy design for dimensional measurement of geometric features using coordinate measuring machine," International Journal of Machine Tools and Manufacture, vol. 37, no. 7, pp. 917-934, 7// 1997.

[22] W.-S. Kim and S. Raman, "On the selection of flatness measurement points in coordinate measuring machine inspection," International Journal of Machine Tools and Manufacture, vol. 40, no. 3, pp. 427-443, 2// 2000.

[23] X. Zhang, X. Jiang, and P. J. Scott, "Minimum zone evaluation of the form errors of quadric surfaces," Precision Engineering, vol. 35, no. 2, pp. 383-389, $2011 / 04 / 01 / 2011$

[24] Y. Arezki, X. Zhang, C. Mehdi-Souzani, N. Anwer, and H. Nouira, "Investigation of minimum zone assessment methods for aspheric shapes," Precision Engineering, 2018/02/13/2018.

[25] I. Ainsworth, M. Ristic, and D. Brujic, "CAD-Based Measurement Path Planning for Free-Form Shapes Using Contact Probes," The International Journal of Advanced Manufacturing Technology, vol. 16, no. 1, pp. 23-31, 2000.

[26] D. F. Elkott, H. A. Elmaraghy, and W. H. Elmaraghy, "Automatic sampling for CMM inspection planning of free-form surfaces," International Journal of Production Research, vol. 40, no. 11, pp. 2653 - 2676, 2002.

[27] Y. F. Li and Z. G. Liu, "Method for determining the probing points for efficient measurement and reconstruction of freeform surfaces," Measurement Science and Technology, vol. 14, no. 8, p. 1280, 2003. 
[28] K. D. Summerhays, R. P. Henke, J. M. Baldwin, R. M. Cassou, and C. W. Brown, "Optimizing discrete point sample patterns and measurement data analysis on internal cylindrical surfaces with systematic form deviations," Precision Engineering, vol. 26, no. 1, pp. 105-121, 2002/01/01/2002.

[29] R. Raghunandan and P. V. Rao, "Selection of an optimum sample size for flatness error estimation while using coordinate measuring machine," International Journal of Machine Tools \& Manufacture, vol. 47, no. 3-4, pp. 477-482, 2007.

[30] M. A. Badar, S. Raman, and P. S. Pulat, "Intelligent Search-Based Selection of Sample Points for Straightness and Flatness Estimation," Journal of Manufacturing Science \& Engineering, vol. 125, no. 2, pp. 263-271, 2003.

[31] S. M. Obeidat and S. Raman, "An intelligent sampling method for inspecting free-form surfaces," International Journal of Advanced Manufacturing Technology, vol. 40, no. 11-12, pp. 1125-1136, 2009.

[32] Y. Li and P. Gu, "Automatic localization and comparison for free-form surface inspection," Journal of Manufacturing Systems, vol. 25, no. 4, pp. 251-268, 2006.

[33] M.-W. Cho, H. Lee, G.-S. Yoon, and J. Choi, "A feature-based inspection planning system for coordinate measuring machines," (in English), The International Journal of Advanced Manufacturing Technology, vol. 26, no. 9-10, pp. 1078-1087, 2005/10/01 2005.

[34] A. Barari, H. A. ElMaraghy, and G. K. Knopf, "Search-Guided Sampling to Reduce Uncertainty of Minimum Deviation Zone Estimation," Journal of Computing and Information Science in Engineering, vol. 7, no. 4, pp. 360-371, 2007.

[35] Y. Yin, M. J. Ren, and L. Sun, "Dependant Gaussian processes regression for intelligent sampling of freeform and structured surfaces," CIRP Annals Manufacturing Technology, 2017.

[36] Y. Yin, M. J. Ren, L. Sun, and L. Kong, "Gaussian process based multi-scale modelling for precision measurement of complex surfaces," CIRP Annals Manufacturing Technology, vol. 65, no. 1, pp. 487-490, 2016.

[37] P. Pedone, G. Vicario, and D. Romano, "Kriging - based sequential inspection plans for coordinate measuring machines," Applied Stochastic Models in Business \& Industry, vol. 25, no. 2, pp. 133-149, 2009.

[38] R. Ascione, G. Moroni, S. Petrò, and D. Romano, "Adaptive inspection in coordinate metrology based on kriging models," Precision Engineering, vol. 37, no. 1, pp. 44-60, 2013.

[39] M. J. Ren, C. F. Cheung, and L. B. Kong, "A bidirectional curve network based sampling method for enhancing the performance in measuring ultra-precision freeform surfaces," Precision Engineering, vol. 37, no. 2, pp. 345-352, 2013/04/01/2013.

[40] M. Yu, Y. Zhang, Y. Li, and D. Zhang, "Adaptive sampling method for inspection planning on CMM for free-form surfaces," The International Journal of Advanced Manufacturing Technology, vol. 67, no. 9, pp. 1967-1975, 2013/08/01 2013.

[41] L. Sun, M. Ren, and Y. Yin, "Domain-specific Gaussian process-based intelligent sampling for inspection planning of complex surfaces," International Journal of Production Research, vol. 55, no. 2, pp. 1-15, 2017.

[42] P. Pedone and D. Romano, "Designing small samples for form error estimation with coordinate measuring machines," Precision Engineering, vol. 35, no. 2, pp. $262-270,2011 / 04 / 01 / 2011$.

[43] M. A. Badar, S. Raman, and P. S. Pulat, "Experimental verification of manufacturing error pattern and its utilization in form tolerance sampling," International Journal of Machine Tools and Manufacture, vol. 45, no. 1, pp. 63-73, 1// 2005.

[44] R. K. Leach, C. W. Jones, B. Sherlock, and A. Krysinski, "The high dynamic range surface metrology challenge," presented at the 28th Annual Meeting of the American Society for Precision Engineering, St. Paul, Minnesota, USA, 2013.

[45] W. Li, S. Xu, G. Zhao, and L. P. Goh, "Adaptive knot placement in B-spline curve approximation," Computer-Aided Design, vol. 37, no. 8, pp. 791-797, 2005/07/01/2005.

[46] S. Spiriti, R. Eubank, P. W. Smith, and D. Young, "Knot selection for least-squares and penalized splines," Journal of Statistical Computation and Simulation, vol. 83, no. 6, pp. 1020-1036, 2013/06/01 2013.

[47] T. Schütze and H. Schwetlick, "Bivariate Free Knot Splines," BIT Numerical Mathematics, vol. 43, no. 1, pp. 153-178, 2003/03/01 2003.

[48] T. Tjahjowidodo, V. Dung, and M. Han, "A fast non-uniform knots placement method for B-spline fitting," in 2015 IEEE International Conference on Advanced Intelligent Mechatronics (AIM), 2015, pp. 1490-1495.

[49] Geometrical product specifications (GPS) -- Geometrical tolerancing -- Tolerances of form, orientation, location and run-out, 2012.

[50] C. de Boor, A Practical Guide to Splines (Applied Mathematical Sciences). Springer, 2001.

[51] J. Wang, L. Pagani, R. K. Leach, W. Zeng, B. M. Colosimo, and L. Zhou, "Study of weighted fusion methods for the measurement of surface geometry," Precision Engineering, vol. 47, pp. 111-121, 2016.

[52] R. M. Barker, M. G. Cox, A. B. Forbes, and P. M. Harris, "Best Practice Guide No. 4 Software Support for Metrology: Discrete modelling and experimental data analysis," in "Technical report, National Physical Laboratory," Teddington, UK2004.

[53] G. H. Golub and V. Pereyra, "The Differentiation of Pseudo-Inverses and Nonlinear Least Squares Problems Whose Variables Separate," Siam Journal on Numerical Analysis, vol. 10, no. 2, pp. 413-432, 1973.

[54] X. Zhao, C. Zhang, B. Yang, and P. Li, "Adaptive knot placement using a GMM-based continuous optimization algorithm in B-spline curve approximation," Computer-Aided Design, vol. 43, no. 6, pp. 598-604, 2011/06/01/2011.

[55] J. Pittman, "Adaptive Splines and Genetic Algorithms," Journal of Computational \& Graphical Statistics, vol. 11, no. 3, pp. 615-638, 2002.

[56] M. M. Dowling, P. M. Griffin, and C. Zhou, "Statistical Issues in Geometric Feature Inspection Using Coordinate Measuring Machines," Technometrics, vol. 39, no. 1, pp. 3-17, 1997.

[57] J. P. C. Kleijnen, "Application-Driven Sequential Designs for Simulation Experiments: Kriging Metamodelling," Journal of the Operational Research Society, vol. 55 , no. 8, pp. 876-883, 2004

[58] G. Gutin, A. Yeo, and A. Zverovich, "Traveling salesman should not be greedy: domination analysis of greedy-type heuristics for the TSP," Discrete Applied Mathematics, vol. 117, no. 1, pp. 81-86, 2002.

[59] T. Dokken, T. Lyche, and K. F. Pettersen, "Polynomial splines over locally refined box-partitions," Computer Aided Geometric Design, vol. 30, no. 3, pp. 331$356,2013 / 03 / 01 / 2013$.

[60] J. Wang, "Sampling for the Measurement of Structured Surfaces," Doctoral, School of Computing and Engineering, University of Huddersfield, 2012.

[61] W. L. Li, A. Wu, Z. C. Li, G. Zhang, and W. Y. Yu, "A new calibration method between an optical sensor and a rotating platform in turbine blade inspection," Measurement Science \& Technology, vol. 28, no. 3, p. 035009, 2017.

[62] C. E. Rasmussen and C. Williams, Gaussian Processes for Machine Learning. the MIT Press, 2006

[63] W. Ma and J.-P. Kruth, "Parametrization of randomly measured points for least squares fitting of B-spline curves and surfaces," Computer-Aided Design, vol. 27, pp. 663-675, 1995. 